\title{
Esophageal Involvement in Epidermolysis Bullosa Dystrophica: Clinical and Roentgenographic Manifestations
}

\author{
Farooq P. Agha, ${ }^{1}$ Isaac R. Francis, ${ }^{1}$ and Charles N. Ellis ${ }^{2}$ \\ Departments of ${ }^{1}$ Radiology and ${ }^{2}$ Dermatology, University Hospital, University of Michigan Medical Center, Ann Arbor, \\ Michigan, USA
}

\begin{abstract}
Epidermolysis bullosa is a rare hereditary mechanobullous skin disorder. Four patients with esophageal involvement are reported. These cases and review of reported cases of epidermolysis bullosa dystrophica-recessive (EBD-R) showed distinct clinical and roentgenographie features of esophageal involvement: diffuse inflammatory changes, motility disorders, small blisters or bullae seen as constant nodular-filling defects, esophageal ulcers, scars, pseudodiverticula, transverse and circumferential webs, various size strictures, shortening of the esophagus with development of traction hiatal hernia and gastroesophageal reflux, perforation, and complete obstruction of the esophageal lumen. Dysphagia is reversible when caused by bullae or webs and permanent when due to cicatrizing strictures. The strictures may remain unchanged in size for many years despite variations in dysphagic symptoms.
\end{abstract}

Key words: Esophagus, inflammation - Esophageal stricture, web, ulcer, perforation, obstruction Epidermolysis bullosa, esophageal involvement.

Epidermolysis bullosa is a rare genetic skin disease distinct from other bullous dermatoses. It encompasses a group of hereditary disorders characterized by bullae and subsequent erosions in the areas of mechanical irriation. Two major groups are defined; a dystrophic (scarring) group and a nondystrophic group. Subgroups are identified according to the mode of inheritance, age of onset, pathology, and clinical course [1]. The dystrophic subgroups are epidermolysis bullosa dystrophica-

Address reprint requests to: Farooq P. Agha, M.D., Department of Radiology, Box 13, University Hospital, University of Michigan, Ann Arbor, MI 48109, USA dominant inheritance (EBD-D), epidermolysis bullosa dystrophica-recessive inheritance, (EBD$\mathrm{R}$ ), and epidermolysis bullosa-acquisita (EBD-A). The nondystrophic group includes epidermolysis bullosa simplex, recurrent bullous eruptions of feet and hands (Weber's and Cockayne's syndromes), and epidermolysis bullosa letalis (junctional bullous dermatosis).

The EBD-R also referred to as polydysplastic form usually manifests bullae in the perinatal period. Bullae may involve the skin, mucous membranes, and other organs lined by stratified squamous epithelium. In severe cases, congenital localized absence of the skin may accompany the eruptions [2]. Only patients with EBD-R develop clinically significant esophageal involvement. In patients who survive past early childhood, esophageal involvement is exceedingly rare. Gedde-Dahl [3] found only 3 out of 55 patients with EBD-R showing esophageal involvement. Approximately 60 cases with esophageal involvement in EBD-R have been recorded in the literature [3-14]. Dysphagia is a major symptom as the lesions progress from bullae to erosions, ulcers, scars, and to strictures. Despite variable dysphagic symptoms, strictures remain unchanged in size for many years.

We report 4 cases of EBD-R with esophageal involvement. Clinical and roentgenographic features with particular reference to distinct changes in the esophagus in these cases and previously reported cases from the literature review, form the basis of this report.

\section{Case Reports}

Case 1

A 32-year-old man had a known diagnosis of polydysplastic epidermolysis bullosa dating from birth. His disease had been characterized by recurrent blisters over his trunk and extremi- 

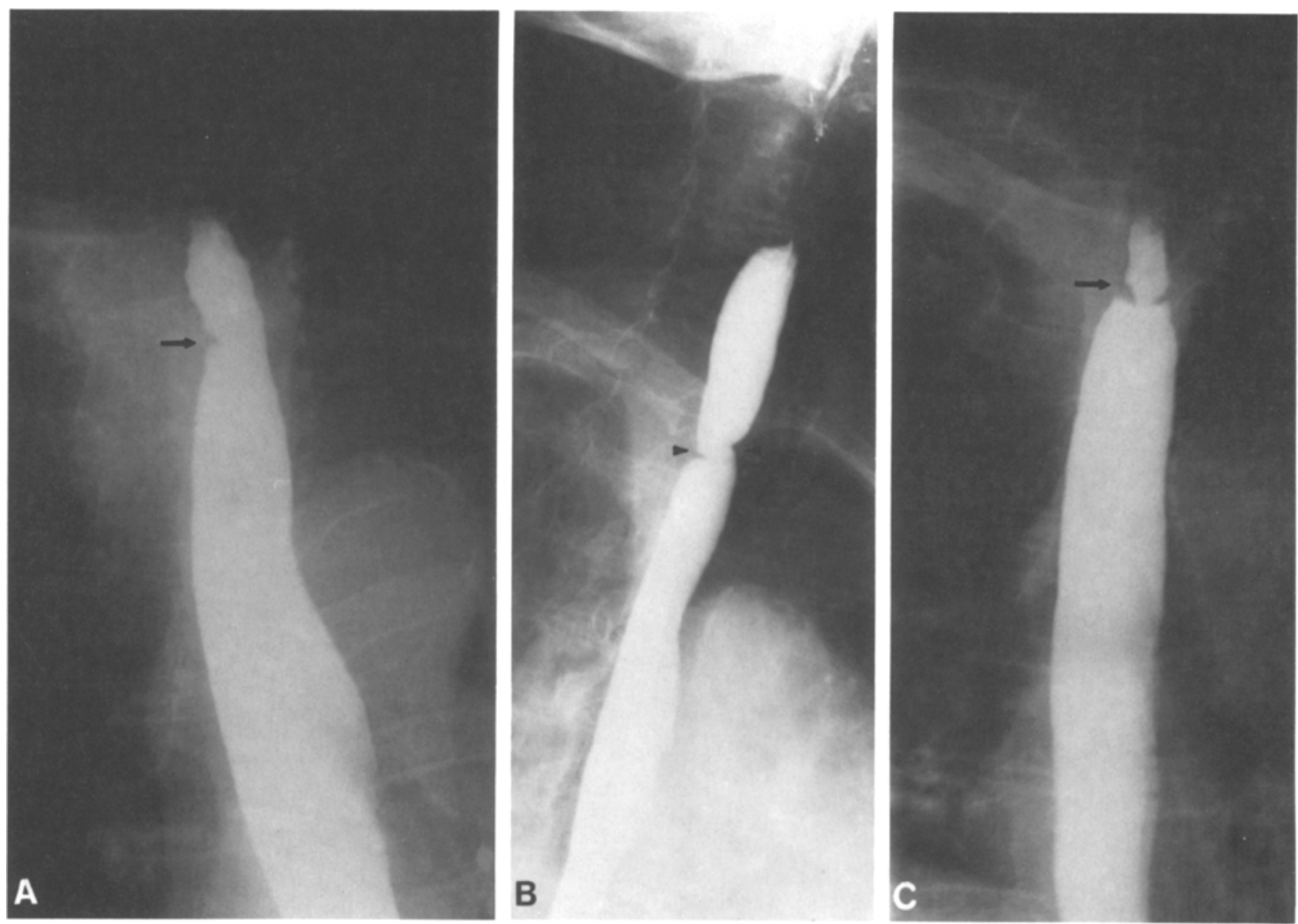

Fig. 1. Case 1. A Esophagogram of April 1976 showing a small transverse web in the upper thoracic esophagus (arrow). B Esophagogram of March 1978 demonstrates development of a circumferential web (arrowhead) causing marked luminal narrowing (luminal diameter, $6 \mathrm{~mm}$ ). C Barium study from April 1979 reveals a tight short stricture (arrow) with luminal diameter of $3 \mathrm{~mm}$

ties. Secondary to this he had developed cutaneous scarring and flexion contractures of his hands and feet, deformed teeth with frequent caries, and occasional pharyngeal bullac. At age 26 years he first experienced mild dysphagia. He was referred to the out-patient dermatology clinic of West Virginia University Hospital. A barium swallow in April 1976 (Fig. 1 A) showed a small transverse web in the upper esophagus. Symptoms of dysphagia gradually increased over the next 2 years. An esophagogram in March 1978 (Fig. 1B) revealed a circumferential web in the proximal esophagus with an inner diameter of $6 \mathrm{~mm}$. Esophagoscopy using an infant endoseope revealed atrophic and friable mucosa. A circumferential web was encountered $22 \mathrm{~cm}$ from the upper alveolar ridge. Using extreme caution the endoscopist dilated the esophagus with bougies to number 38 French dilator. A year later the patient presented with extreme dysphagia. A $3 \mathrm{~mm}$ stricture (Fig. 1C) had developed at the site of previous circumferential web. Apparently the trauma due to dilatation triggered progression of cicatrization. Since then he had required repeated bougienage. In addition he had received $5 \mathrm{mg}$ prednisone 4 times daily. With treatment he has been able to eat a normal diet. He had no further problems with his cutaneous and pharyngeal bullae since starting prednisone. When last seen in August 1979 he was eating without dysphagia.

\section{Case 2}

A 28-year-old woman with known diagnosis of epidermolysis bullosa dystrophica since birth first complained of dysphagia at 12 years of age. The dysphagia became progressively worse over the years. She was treated on and off by a local dermatologist. In 1971 she was admitted to Bird S. Coler Hospital, New York City, for evaluation of marked weakness. On examination an emaciated woman weighing 63 lbs with severe flexion contracture deformities of both extremities, atrophic scarring of the skin, and dystrophic nails was noted. She had erosions in her groin and a few blisters in her upper chest wall. Due to microstomia the opening of the mouth was limited to $2.5 \mathrm{~cm}$ and she could not protrude her tongue more than $2 \mathrm{~cm}$. The oral mucosa was unusually raw and congested. A barium swallow obtained during her work-up revealed 2 strictures in the upper esophagus (Fig. 2). The upper stricture measured $3 \mathrm{~cm}$ in length with a $2-3 \mathrm{~mm}$ lumen. A small ulcer was seen projecting posteriorly from the midpoint of this stricture. The cervical esophagus proximal to the stricture was minimally dilated with diminished peristaltic activity. The second stricture in the mid esophagus measured $1.5 \mathrm{~cm}$ in length with a maximum luminal diameter of $6 \mathrm{~mm}$. The mucosa was smooth. No hiatal hernia or gastroesophageal reflux was noted. An endo- 


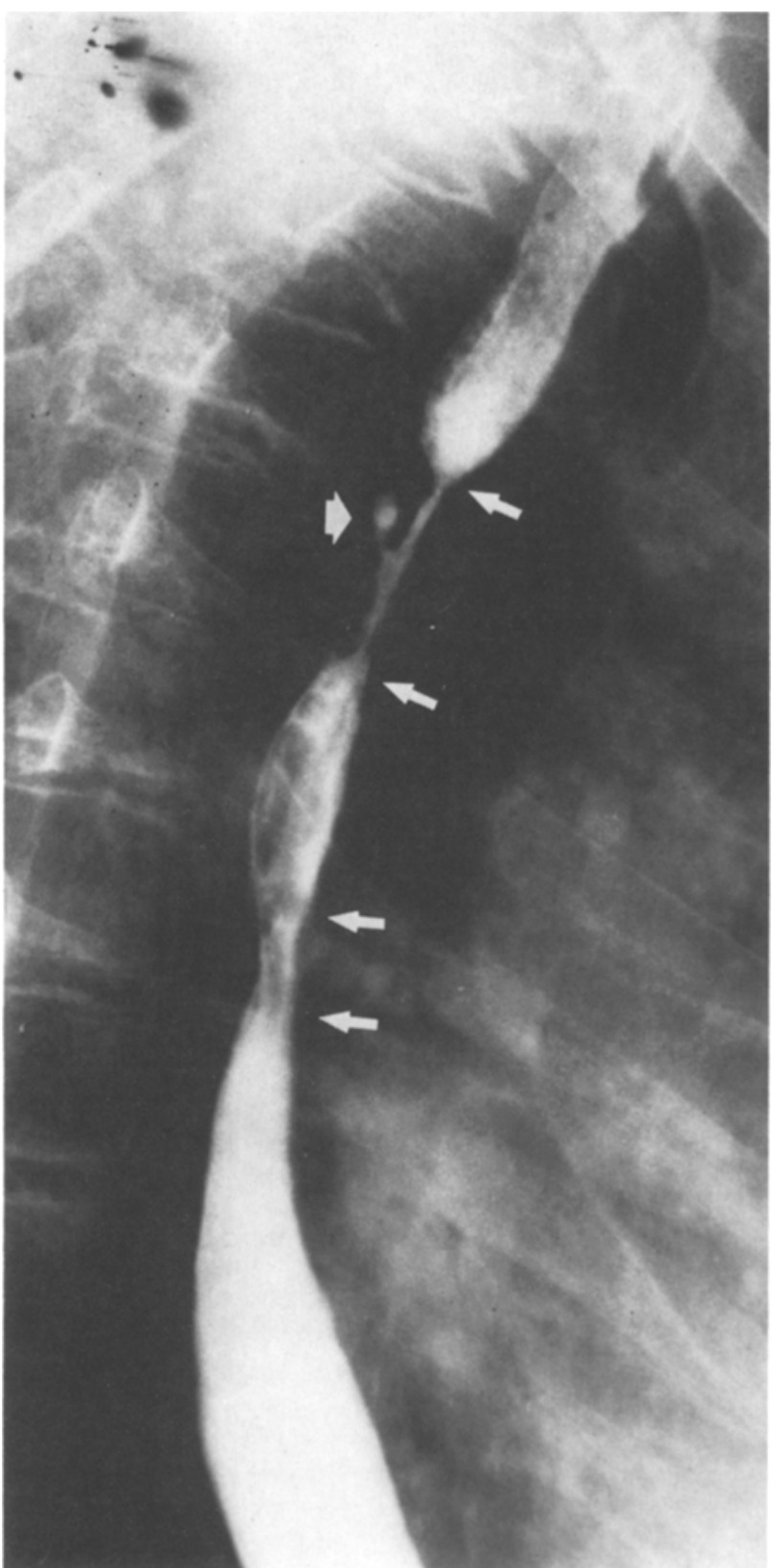

Fig. 2. Case 2. Esophagogram showing 2 strictures (arrows) in the proximal esophagus. The upper stricture is $3 \mathrm{~cm}$ long with a luminal diameter of $2-3 \mathrm{~mm}$. An ulcer is seen projecting posteriorly from the midportion of this stricture (broad arrow). Minimal prestenotic dilatation of proximal esophagus is present. The lower stricture is $1.5 \mathrm{~cm}$ long with luminal diameter of $6 \mathrm{~mm}$

scopic examination was limited because of microstomia, blisters, and raw mucosa in the pharynx. The patient was treated with steroids and general supportive therapy including several blood transfusions. When last seen in 1974 she was eating liquid and semisolid foods with less dysphagia.

\section{Case 3}

A 34-year-old woman had EBD-R since birth in 1946. There was no family history of this disease. She was examined at irregular intervals by a dermatologist and received therapy of various kinds, both topical and systemic. At age 5 years she first experienced dysphagia which became progressively worse over the years. In September 1963 a barium swallow revealed complete obstruction of the esophagus at the level of the aortic arch. She underwent repeated esophageal dilatation during the next 2 years and eventually a number 43 French size plummer dilator could be passed without difficulty. In 1966 she experienced another acute episode of dysphagia. A barium swallow (Fig. 3A) revealed several small constant nodular-filling defects consistent with esophageal bullae, diminished peristaltic activity, spasm, and few areas of mucosal scarring. The dysphagia improved on steroid therapy. Recurrent buccal and pharyngeal bullae led to intraoral scarring and obliteration of the mandibular mucobuccal folds. In 1970 an operation was performed to release buccal scarring and lower the floor of her mouth. This corrected mandibular-alveolar ridge deficiency and she was able to open her mouth and eat more easily; however, with increased ability to eat solid foods she noted increasing dysphagia. A barium swallow and cineradiographic study in 1970 (Fig. 3B) revealed diffuse narrowing of the entire esophagus except for the ampullary portion. No ulceration was identified. Some stripping wave activity was noted in the proximal esophagus. She underwent esophageal dilations beginning with number 23 French dilator and progressing to number 39 French. When the dilator was removed at the last procedure a cast of esophageal mucosa was avulsed. The dilatation was discontinued and in April 1971 she underwent a left colonic transplant with esophagogastrocolic anastosmosis, pyloroplasty, temporary gastrostomy, and feeding jejunostomy. Before and after surgery she received corticosteroids and she has been maintained on low-dosage therapy thereafter. The postoperative period was complicated by extensive loss of epidermis from the chest and abdomen brought about by the surgical preparation of the operative field. However the skin healed readily on exposure to air, sterile petroleum jelly, and gentamycin ointment. The patient was able to swallow food by the 8 th postoperative day. When last seen in June 1980, 9 years after her operation, she was eating without dysphagia and the colon transplant was functioning well (Fig. 3C).

\section{Case 4}

A 16-year-old male with congenital EBD-R was admitted to Mott's Childrens Hospital, Ann Arbor, in October 1981 for general evaluation and initiation of Dilantin ${ }^{\circledR}$ (diphenylhydantoin) therapy in the hope of decreasing the formation of new blisters. Five days prior to admission he spat up a small amount of blood secondary to rupture of a mucosal blister.

He was a product of normal gestation and, according to his mother, was born "without skin." $\mathrm{He}$ is the only abnormal child of 4 children with no family history of EBD on either parent's side. Over the years he has developed severe flexion contracture deformities of his extremities, syndactyly of his fingers and toes, microstomia due to recurrent oropharyngeal blistering and scarring, extensive dental caries, and phimosis.

In 1979 he complained of dysphagia; a barium swallow (Fig. 4A) revealed a high esophageal stricture. There was no obstruction to the flow of barium. He was maintained on liquid and semisolid diet. Recently he has been eating less due to oropharyngeal discomfort and has lost some weight.

Physical examination at the time of admission revealed a small-stature male with diffuse muscle wasting weighing $34 \mathrm{lbs}$. His skin revealed fairly extensive blistering, erosions, and scarring. The extremities showed flexion contractures, syndactyly of fingers and toes, and dystrophies of nails. He was unable to open his mouth more than $3 \mathrm{~cm}$ and could not protrude 

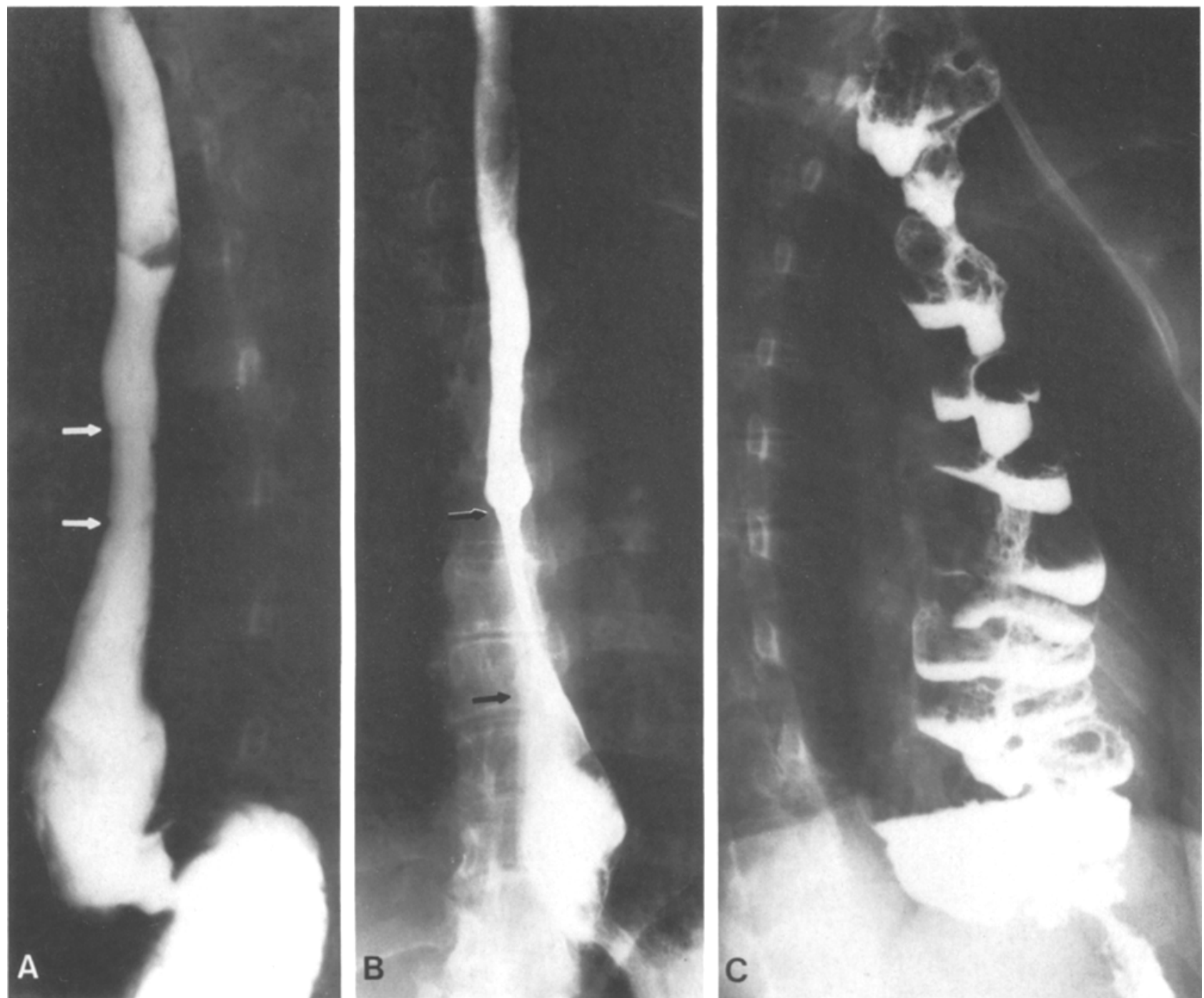

Fig. 3. Case 3. A Esophagogram of February 1966 during an acute episode of dysphagia associated with oropharyngeal bullae showing several constant nodular-filling defects representing bullae (arrows show 2 defects only). B Esophagogram of November 1970 showing diffuse narrowing of the entire esophagus with mucosal irregularities due to scarring. A 5-cm long narrow stricture was noted in the distal esophagus (arrows) with development of a traction hiatal hernia. C Barium study from June 1980 showing status after colonic transplant with esophagogastrocolic anastomosis. This colonic replacement of esophagus was performed in April 1971 and is functioning well 9 years later

his tongue. A $5 \mathrm{~mm}$ erosion on the dorsum of the tongue and an $8 \mathrm{~mm}$ erosion on the left side of hard palate were noted. Extensive dental caries with several previous dental extractions were noted. Chest and abdominal examination was unremarkable.

Laboratory studies revealed chronic hypochromic microcytic anemia. A barium swallow (Fig. 4B) revealed a high esophageal stricture with mucosal irregularities secondary to scarring, a small hiatal hernia, and gastroesophageal reflux. There was no change in this study when compared with that done 2 years previously. The patient was treated with steroids. No esophageal dilatation was attempted. Dysphagia improved and he was maintained on liquid and semisolid diet. When last seen in June 1982 he had less dysphagia. However, anemia was refractory to conventional treatment and had worsened.

\section{Discussion}

Kobner in 1886 [15] proposed the name generally accepted later as "epidermolysis bullosa hereditaria." Siemens in 1921 [16] introduced analysis of recessive Mendelian inheritance in this disease and was able to show a close association between dominant inheritance, absence of dystrophies, and intraepidermal localization of blisters on the one hand and recessive inheritance, dystrophies, and subepidermal bullae formation on the other. From then on patients showing nail dystrophies, skin atrophies, and scarring have been classified as 


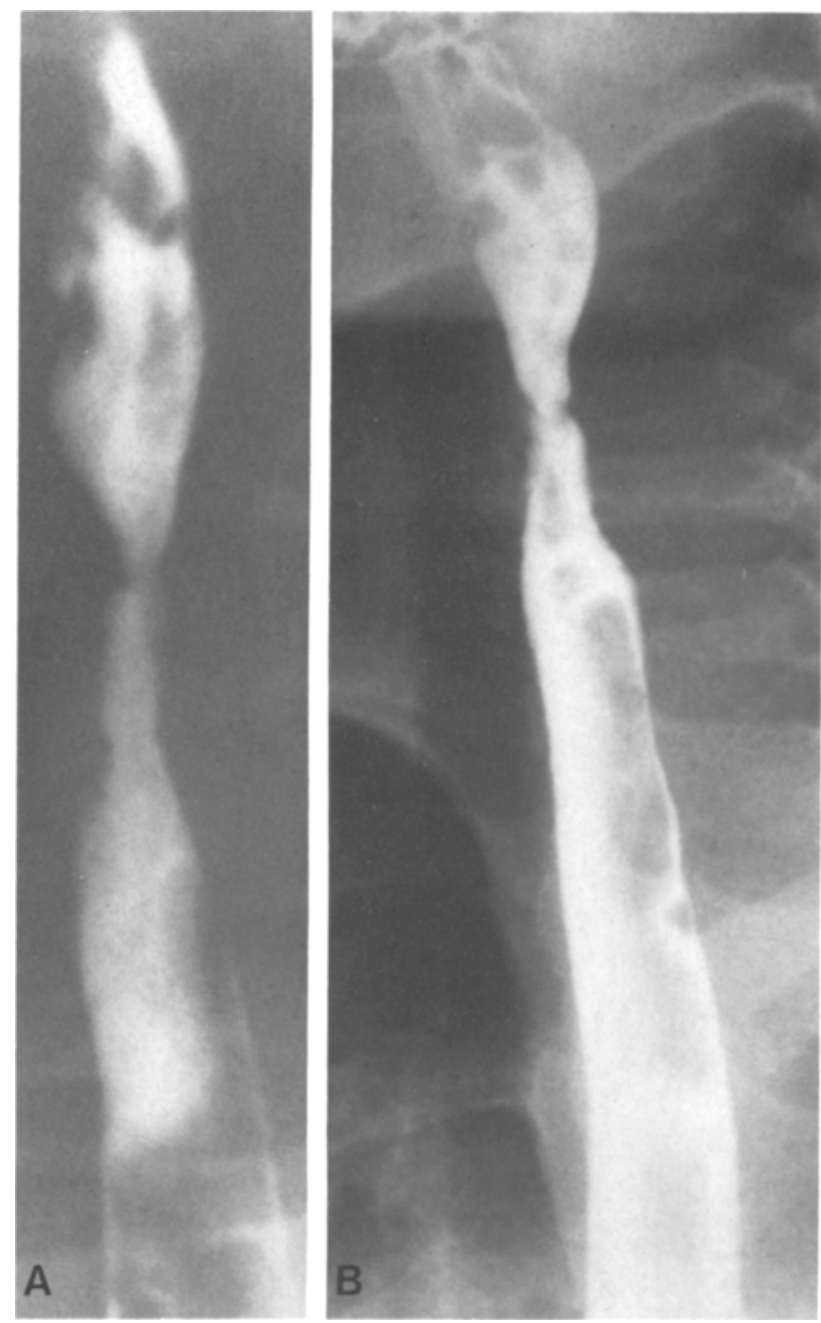

Fig. 4. Case 4. A Esophagogram of August 1979 showing a high esophageal stricture with mucosal irregularities. B Barium study of June 1981 showing no change in the size and shape of the stricture, although the symptoms of dysphagia had increased

having EBD-R and those lacking signs of dystrophies as having epidermolysis bullosa simplex.

\section{Pathogenetic Considerations}

Electron-microscopic examination of blistered and unblistered skin shows absence of derangement of connective-tissue fibrils and separation in the area immediately below the basal lamina. In all dystrophic forms of epidermolysis bullosa the dermalepidermal split is below the basal lamina lying in the upper part of dermis, therefore, the lamina densa forms the bulla's roof and the dermal tissue forms the bulla's base [16-19]. Anchoring fibrils are markedly diminished or absent and may contribute to the weakness in structural attachment of lamina densa to the dermis [20].
Collagenase activity of skin from friction bullae produced in patients with EBD-R is approximately 6 times greater than that found in normal skin. Increased collagenolytic activity of epidermis and papillary dermis due to production of specific collagenase may contribute to bullae formation [21].

\section{Clinical Features}

EBD-R begins at or soon after birth. Bullae may involve the skin, mucous membranes, and other organs lined by stratified squamous epithelium such as esophagus, bronchus, and anus. In severe cases congenital localized absence of the skin may accompany the eruptions [2]. Scarring frequently results in loss of hair, nails become dystrophic, and are subsequently lost. When cutaneous scarring is severe and occurs near a joint, it may cause flexion contractures; syndactyly may appear as repeated bullae and cicatrization encase the phalanges and toes in scar tissue.

Subsequently characteristic club-shaped deformities of hand and feet develop. Milia are common in EBD-R. Only patients with EBD-R develop clinically apparent esophageal involvement. Dysphagia is the major presenting symptom as the disease process involves the esophagus. Altough reversible early on, dysphagia becomes permanent as the inflammatory lesions are replaced by scar formation and permanent strictures. Dysphagia usually starts insidiously in the first decade of life, but delay until the fifth decade has been reported. Fifty percent of the patients reviewed by Nix and Christianson [7] showed dysphagia for more than 20 years. Three out of 4 of the patients reported here had symptoms of dysphagia for at least 10 years. The esophageal lesions that start by adolescence may contribute to patient's demise through inanition or aspiration [7, 14].

\section{Roentgenographic Features}

Barium studies of the esophagus are an effective way of showing esophageal lesions. Steurer in 1921 [22] reported narrowing of the esophageal lumen (a fluoroscopic observation). The first radiologic report of esophageal involvement was by Stout in 1929 [23]. Radiographic features of esophageal involvement may be variable at different stages of the disease and can be best summarized as follows.

Diffuse Inflammatory Changes. Nonspecific inflammatory changes may be seen early in the course of the disease, manifested as mucosal edema, and areas of inconstant spasm. Superficial 
erosions or ulcerations secondary to eruption of bullae may be seen.

Disordered Motility. In early stages, motility is not affected. Primary stripping wave is seen without impairment. As diffuse inflammatory changes progress to scarring, motility disturbances may develop. Peristalsis proximal to the stenotic segment is usually diminished in activity.

Bullae, Ulcers, and Mucosal Scarring. Small constant nodular-filling defects representing bullae (Fig. 3 A) may be seen if the barium studies are done early in the course of the disease or during a recurrent bullous episode. Occasionally ulcers can be demonstrated (Fig. 2). These heal very slowly because of extensive surrounding scar tissue. Mucosal irregularities due to scarring are commonly seen in patients during quiescent stages of the disease (Fig. 4A). Occasionally a pseudodiverticulum may develop as scarring and healing of ulcer progresses.

Webs. Postinflammatory webs may develop (Fig. 1 A) as healing continues, starting as a small transverse web. As scarring progresses transverse webs may form circumferential webs by approximation of opposite walls (Fig. 1 B) with severe dysphagic symptoms. These webs are quite similar to those described in pemphigoid [24].

Strictures. The most common site of involvement of the esophagus is the upper third $(50 \%)$ followed by lower third $(25 \%)$; in $25 \%$ of cases multiple sites are involved. Single or multiple strictures of variable length may be seen. The average length of strictures is $2-6 \mathrm{~cm}$. However, longer strictures as well as the entire esophagus may be narrowed (Fig. 3B) with areas of variable luminal stenosis. There is usually some prestenotic dilatation. At the proximal margin of the stricture, abrupt transition from normal to abnormal mucosa may be seen; gradual tapering with smooth or scarred mucosa is seen distally. Once strictures are formed, they are permanent. They may remain unchanged in size and length for many years despite variations in dysphagic symptoms. Light trauma induced by solid food or endoscopy may trigger progression of the stricture.

Traction Hiatal Hernia and Gastroesophageal Reflux. As cicatrization progresses, shortening of esophagus may lead to development of traction hiatal hernia and gastroesophageal reflux. Manometric and radiologic studies for gastroesophageal reflux by Orlando et al. [14] failed to support the concept that reflux was the major factor in the production of lower esophageal strictures in EBD.

Complete Obstruction of Esophagus. Complete occlusion of the lumen of the esophagus may occur due to diffuse bullous and ulcerative lesions of the esophagus, stenotic lesions $2-6 \mathrm{~cm}$ in length, and multiple areas of stricture formation. A markedly narrowed stricture may trap food particles and lead to complete luminal occlusion [7]. Case 3 developed esophageal occlusion due to diffuse bullous disease during an episode of bullous eruptions.

Perforation of Esophagus. Nix et al. [7] reported a 22-year-old man with EBD-R since birth and esophageal lesions since 8 years of age, who perforated his esophagus while attempting to eat a toasted cheese sandwich. A stricture trapped food particles causing obstruction, which prevented adequate propulsion of the esophageal contents. The resultant increased intraluminal pressure was sufficient to cause rupture. A 2-cm long tear occurred in the posterior wall of esophagus $5 \mathrm{~cm}$ above the gastroesophageal junction. The patient died $36 \mathrm{~h}$ after admission to the hospital despite aggressive therapeutic measures.

\section{Complications of Esophageal Lesions}

The most common complication of EBD-R of the esophagus is the lodging of food in the esophagus. This can usually be relieved by endoscopy. Serious hemorrhage has occurred, but there have been no reported fatalities [7]. Carcinoma developing at the cardia and extending into the esophagus has been reported in a 38-year-old woman with severe EBD of the esophagus [25]. Pulmonary disease was a predominant feature in a 32-year-old woman who had had EBD since childhood and esophageal stenosis for 10 years. Repeated aspirations into the right lung of residual food above the esophageal stricture resulted in "dysphagia pneumonitis" [26]. Spontaneous perforation of the esophagus, a fatal complication, has been reported by Nix et al. [7].

Severe malnutrition, refractory anemia, and death from inanition or aspiration pneumonitis typify the disabling nature of EBD-R.

\section{Treatment and Prognosis}

The prognosis of EBD-R of the esophagus is poor. Because of continued odynophagia and inability 
to swallow many foods, severe malnutrition develops. In some susceptible patients the trauma of coarse and hot foods apparently permits the squamous layer to slough off the lamina propria leading to blister and ulcer formation. This mechanical and thermal trauma can be avoided by maintaining patients on a soft bland diet or pureed foods. Endoscopy is only recommended if uncertainty exists concerning the nature or the extent of the disease and prior to any attempt to relieve obstruction by bougineage. Because of the danger of esophageal perforation, endoscopy is not recommended in active stages of the disease and should be performed when the disease is relatively quiescent or under good clinical control with corticosteroids.

Once a stricture is dilated, frequent bougienage is usually required. Prednisone has been effective in controlling blister formation. Absolon [11] reported 2 brothers, aged 8 and 12 years, with progressive dysphagia secondary to esophageal strictures resulting from EBD-R who were successfully treated with total esophageal replacement using right and transverse colon transplants. One patient reported here underwent esophageal replacement using right colon transplant which is functioning well after 10 years. If patients are too weak to undergo surgery or bougienage, a gastrostomy becomes necessary.

Acknowledgment. The authors thank Juanita Cantrell for manuscript preparation.

\section{References}

1. Richter BJ, McNutt NS: The spectrum of epidermolysis bullosa acquisita. Arch Dermatol 115:1325-1328, 1979

2. Bart BJ: Epidermolysis bullosa and congenital localized absence of skin. Arch Dermatol 101:78-81, 1970

3. Gedde-Dahl T Jr: Epidermolysis Bullosa. A Clinical, Genetic and Epidemiologic Study. Baltimore and London: The Johns Hopkins Press, 1971

4. Alpert $\mathrm{M}$ : Roentgen mainfestations of epidermolysis bullosa. AJR 78:66-72, 1957

5. Hadley M, MacDonald AF: Epidermolysis bullosa. $\mathrm{Br} J$ Radiol 33:646-652, 1960

6. Stritzler C: Epidermolysis bullosa, dystrophic type, with stricture of esophagus. Arch Dermatol 81:159, 1960

7. Nix TE, Christianson HB: Epidermolysis bullosa of the esophagus: report of two cases and review of literature. South Med J 58:612-620, 1965
8. Bergenholtz A, Olsson O, Arwill T, Lundstrom NR: Esophageal changes in epidermolysis bullosa hereditaria dystrophica. Pract Otorhinolaryngol 27:219-232, 1965

9. Katz J, Gryboski JD, Rosenbaum HM, Spiro HM : Dysphagia in children with epidermolysis bullosa. Gastroenterology 52:259-262, 1967

10. Becker MH, Swinyard CA: Epidermolysis bullosa dystrophica in children. Radiology 90:124-128, 1968

11. Absolon KB, Finney LA, Waddill GM, Hatchett C: Esophageal reconstruction - colon transplant in two brothers with epidermolysis bullosa. Surgery $65: 832-836,1969$

12. Dupree E, Hodges F Jr, Simon JL: Epidermolysis bullosa of the esophagus. Am J Dis Child 117:349-351, 1969

13. Manier JW, Kaplan AP: Polydysplastic epidermolysis bullosa with esophageal stricture. Gastrointest Endoscopy $19: 19-20,1972$

14. Orlando RC, Bozymski EM, Briggman RA, Bream CA: Epidermolysis bullosa: gastrointestinal manifestations. Ann Intern Med 81:203-206, 1974

15. Kobner $\mathrm{H}$ : Hereditare anlage zur blasenbildung (Epidermolysis bullosa hereditaria). Dtsch Med Wochenschr 21-22, 1886

16. Siemens HW: Zur Klinik, histologic and atiologie der sog. Epidermolysis bullosa traumatica (Bullosis mechanica) mit klinisch-experimentellen studies über die Erzeugung von Reibungsblasen. Arch Dermatol Syphilol 134:454-477, 1921

17. Pearson RW: Studies on pathogenesis of epidermolysis bullosa. J Invest Dermatol 39:551-575, 1962

18. Pearson RW: The mechanobullous diseases (epidermolysis bullosa). In: TB Fitzpatrick, KA Arndt, WH Clark, Jr., AZ Eisen, EJ van Scott, JH Vaughan (eds): Dermatology in General Medicine. New York: McGraw Hill Book Co, 1971, pp 621-643

19. Briggman RA, Wheeler CE: Studies on the pathogenesis of recessive epidermolysis bullosa dystrophica (abstr). $J$ Invest Dermatol 60:109, 1973

20. Briggman RA, Wheeler C: Epidermolysis bullosa dystrophica recessive. A possible role of anchoring fibrils in pathogenesis. J Invest Dermatol 65 :203-211, 1975

21. Eisen AZ: Human skin collagenase; relationship to the pathogenesis of epidermolysis bullosa dystrophica. $J$ Invest Dermatol 52:449-453, 1969

22. Steurer O: Über Beteiligung der Schleimhaut des Mundes und des Speiserohres ber epidermolysis bullosa hereditaria. Arch Ohren Nasen Kehlkopfheilkd 108:11-16, 1921

23. Stout PS: Recurring stricture of the esophagus due to epidermolysis corrosa. Laryngoscope 39:470, 1929

24. Agha FP, Raji MR: Esophageal involvement in pemphigoid: clinical and roentgen manifestations. Gastrointest Radiol 6:109-112, 1982

25. Wettland P, Hovding G: Squamous cell carcinoma in epidermolysis bullosa. Acta Dermatorener 38:27-36, 1956

26. Sedler RL: Dysphagia pneumomitis following epidermolysis bullosa. Br J Dis Chest 49:142-144, 1955

Received: August 24, 1982; accepted: October 27, 1982 